\title{
Site-Fixed Hydroboration of Alkenes under Metal-Free Conditions: Scope and
}

\section{Mechanistic Studies}

\author{
Sida Li, ${ }^{\dagger}, \#$ Chenyang Hu, ${ }^{\&}$ Xin Cui, ${ }^{\dagger}$ Liu Leo Liu, ${ }^{\&, *}$ Lipeng $\mathrm{Wu}^{\dagger, *}$
}

†State Key Laboratory for Oxo Synthesis and Selective Oxidation, Suzhou Research Institute of LICP, Lanzhou Institute of Chemical Physics (LICP), Chinese Academy of Sciences, Lanzhou, 730000 (P. R. China)

${ }^{\#}$ University of Chinese Academy of Sciences, Beijing, 100049 (P. R. China)

${ }^{\&}$ Shenzhen Grubbs Institute and Department of Chemistry, Southern University of Science and Technology, Shenzhen 518055, (P. R. China)

Email: 1iuleoliu@sustech.edu.cn; lipengwu@,licp.cas.cn

\begin{abstract}
An unprecedented and general metal-free hydroboration of alkenes with $\mathrm{BBr}_{3}$ as the boration reagent in the presence of ${ }^{i} \operatorname{Pr}_{2} \mathrm{NEt}$ is reported. The addition of ${ }^{i} \operatorname{Pr}_{2} \mathrm{NEt}$ not only suppresses alkene oligomerization and bromoboration side reactions, but also provides a proton source for hydroboration. More importantly, the site-fixed installation of a boryl group at the original position of the internal double-bond is easily achieved using our strategy as compared with traditional transition-metal-catalyzed hydroboration processes. Preliminary studies on the mechanism revealed a distinct reaction pathway that involves radical species and may operate through frustrated-Lewispair-type single-electron transfer.
\end{abstract}


Organoboronates are synthetically valuable compounds used for a wide range of transformations due to their relative stability, high functional group compatibility, and versatile reactivity. ${ }^{1}$ Boron-containing molecules also play key roles in medicinal chemistry and material science. ${ }^{2}$ Remarkable progress has been made for boronate synthesis through advances in reaction design, catalysis development, and system optimization. ${ }^{3}$ These include, for instance, the transitionmetal-catalyzed boration of (pseudo)halides, ${ }^{4}$ direct C-H boration of (hetero)arenes or alkanes, ${ }^{5}$ and carbene insertion into B-H bonds, etc. ${ }^{6}$ Among them, the catalytic hydroboration of alkenes with borane $\left(\mathrm{H}-\mathrm{BR}_{2}\right)$, is a common route for the construction of $\mathrm{C}-\mathrm{B}$ bonds because it offers advantages of both atom economy and wide substrate availability. ${ }^{7}$ However, despite the great advances in alkene hydroboration, the site-fixed introduction of a boron moiety at the original position of an internal double-bond remains challenging. Traditional catalyst-free hydroboration of internal alkene with $\mathrm{BH}_{3}$ produces internal boronate esters, but with no regioselectivity and low functional group tolerace. ${ }^{8}$ Although improved regioselectivity could be obtained with HBcat, an elevated reaction temperature $\left(100^{\circ} \mathrm{C}\right)$ was required. ${ }^{9}$ It is expected that with transition-metal catalyst, a mild reaction condition could be achieved. However, only a few transition-metals catalyzed site-fixed hydroboration of internal alkenes were known, mainly due to the competitive alkene isomerization which mostly led to the installation of boron moieties at the activated $\alpha$-carbon of an aryl or boryl unit or at the less-hindered terminal position or mixture of isomers (Scheme 1a). In this respect, copper catalyst, literally occurs without competing alkene isomerization, were shown to be a promising candidate as were demonstrated by Hoveyda, ${ }^{10}$ Hartwig, ${ }^{11}$ and Yun (Scheme 1b). ${ }^{12}$ Hence, a general approach that incorporates a boryl group exclusively at the original double-bond position of terminal and internal alkenes remains elusive and more work are needed.

Apart from rational ligand design, system development and chose of suitable transition metals, we envision that another possible strategy to avoid double-bond isomerization, is to use a boron reagent that does not containing or produce $\mathrm{B}-\mathrm{H}$ species during the reaction. ${ }^{13}$ Noting the emerging application of $\mathrm{BX}_{3}(\mathrm{X}=\mathrm{Cl}, \mathrm{Br})$ in the intramolecular borylative cyclization ${ }^{14}$ and $\mathrm{C}-\mathrm{H}$ bond borations, ${ }^{15}$ we decided to explore the possibility of using $\mathrm{BX}_{3}$ as alkene hydroboration reagent. Indeed, the reaction of $\mathrm{BBr}_{3}$ with alkynes and allenes has been reported, which, however, resulted in syn addition of the $\mathrm{B}-\mathrm{Br}$ bond to the unsaturated bonds (known as the bromoboration reaction). ${ }^{16}$ In the presence of stoichiometric amount of silanes, the reaction of $\mathrm{BBr}_{3}$ with alkene was achieved 
via $\mathrm{HBBr}_{2}$, which was formed in-situ. ${ }^{17}$ An substrate-limited classic electrophilic boration of terminal alkenes with $\mathrm{BBr}_{3}$ in the presence of 2,6-disubstituted pyridines to vinyl boronate esters was reported. ${ }^{18}$ Thus, there is no general system available yet for the application of $\mathrm{BBr}_{3}$ in alkene hydroboration given the challenges of chemoselectivity, functional group tolerance, and general substrate scope. Herein, we report our finding of a mechanism distinct utilization of $\mathrm{BBr}_{3}$ as hydroboration reagent of a wide range of alkene under metal-free conditions. More importantly, using our strategy, the exclusive installation of a boron moiety at the original double-bond position of internal alkenes is achieved (Scheme 1c). Studies on the mechanism indicate a reaction pathway that might operate via frustrated Lewis pair (FLP)-type single-electron transfer. ${ }^{19}$

a) General selectivity in transition-metal catalyzed alkene hydroboration

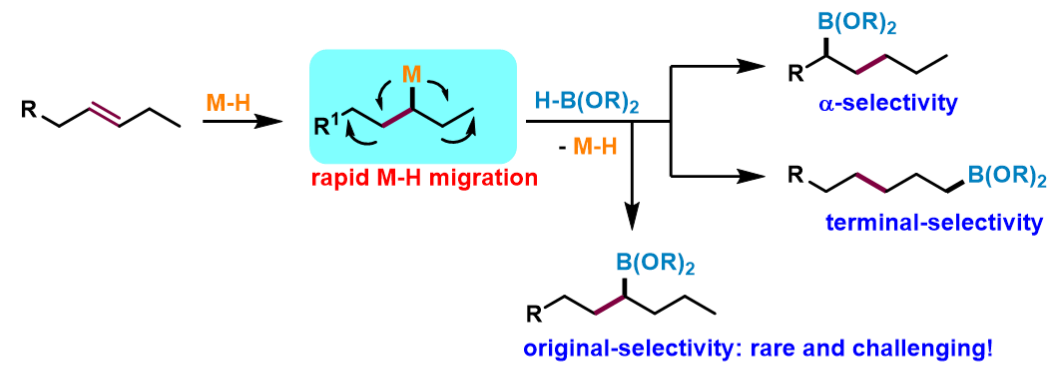

b) Copper-catalyzed site-fixed hydroboration of internal alkene

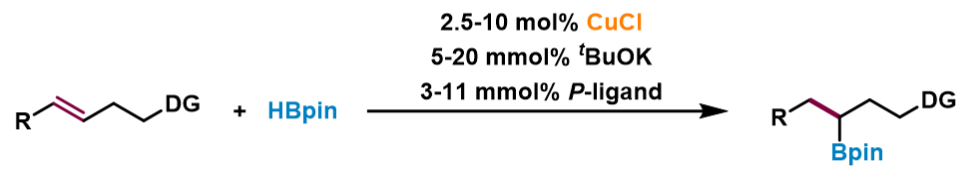

c) Site-fixed alkene hydroboration using $\mathrm{BBr}_{3} /$ amine system - this work
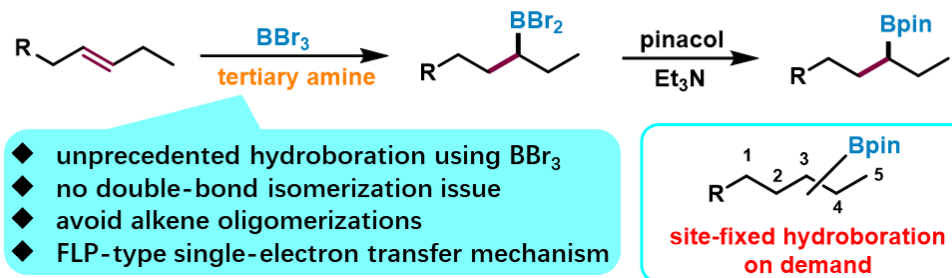

Scheme 1. Site-Fixed Hydroboration of Alkenes: Challenges and Our Strategy.

To apply $\mathrm{BBr}_{3}$ as an efficient boron source in alkene hydroboration, we first need to prevent the alkene oligomerization and bromoboration side reactions. We considered the addition of an amine source would tune the acidity of $\mathrm{BBr}_{3}$. In addition, it is known that boron-containing Lewis acids can be used to abstract a hydrogen atom from the $\alpha$-position of a tertiary alkylamine $;^{20}$ hence, it is expected that the addition of a tertiary amine will not only tune the acidity of the system but also provide a proton source for the hydroboration. To test our hypothesis, the reaction of styrene (0.2 mmol) with $\mathrm{BBr}_{3}(1.0 \mathrm{M}$ in dichloromethane (DCM), 1.5 equiv.) and tertiary amine (3 equiv.) 
was selected as a model. However, when the reaction was conducted with $\mathrm{Et}_{3} \mathrm{~N}, \mathrm{Bn}_{3} \mathrm{~N}$, or ${ }^{n} \operatorname{Pr}_{3} \mathrm{~N}$ at room temperature for $12 \mathrm{~h}$, almost no conversion was observed (Table 1, entries 1-3). As expected, without tertiary amine, styrene dimerization product $\mathbf{5 a}$ and its boration product $\mathbf{3 a}$ with their isomers were obtained in around $8 \%$ yield with no detection of any styrene left on GC/MS which indicated that the rest styrene might be oligomerized (entry 4) (formation of product 3a, 5a or their isomers was unambiguous confirmed by the GC-MS spectra, please see the supporting information GC-MS spectra section); this was also the case when $\mathrm{Ph}_{3} \mathrm{~N}$ was added (entry 5). Using 2,6-lutidine as the amine source, only $25 \%$ of the classic electrophilic boration product $4 \mathbf{a}$ was produced (entry 6). Interestingly, when ${ }^{i} \operatorname{Pr}_{2} \mathrm{NEt}$ was added, $80 \%$ of the target alkyl boronate ester was observed with no detectable side products $\mathbf{3 a}-\mathbf{5 a}$ (entry 7). Changing the solvent from DCM to toluene, hexane, or dichloroethane (DCE), or replacing $\mathrm{BBr}_{3}(1.0 \mathrm{M}$ in $\mathrm{DCM})$ with $\mathrm{BBr}_{3} \cdot \mathrm{SMe}_{2}$ all gave inferior results (entries 8-11). Interestingly, we found that our strategy also worked for $\mathrm{BCl}_{3}$, in which case $75 \%$ of 2 a was obtained (entry 12 ).

Table 1. Effect of Amine and Solvent in the Hydroboration of Styrene with $\mathrm{BBr}_{3}{ }^{\mathrm{a}}$

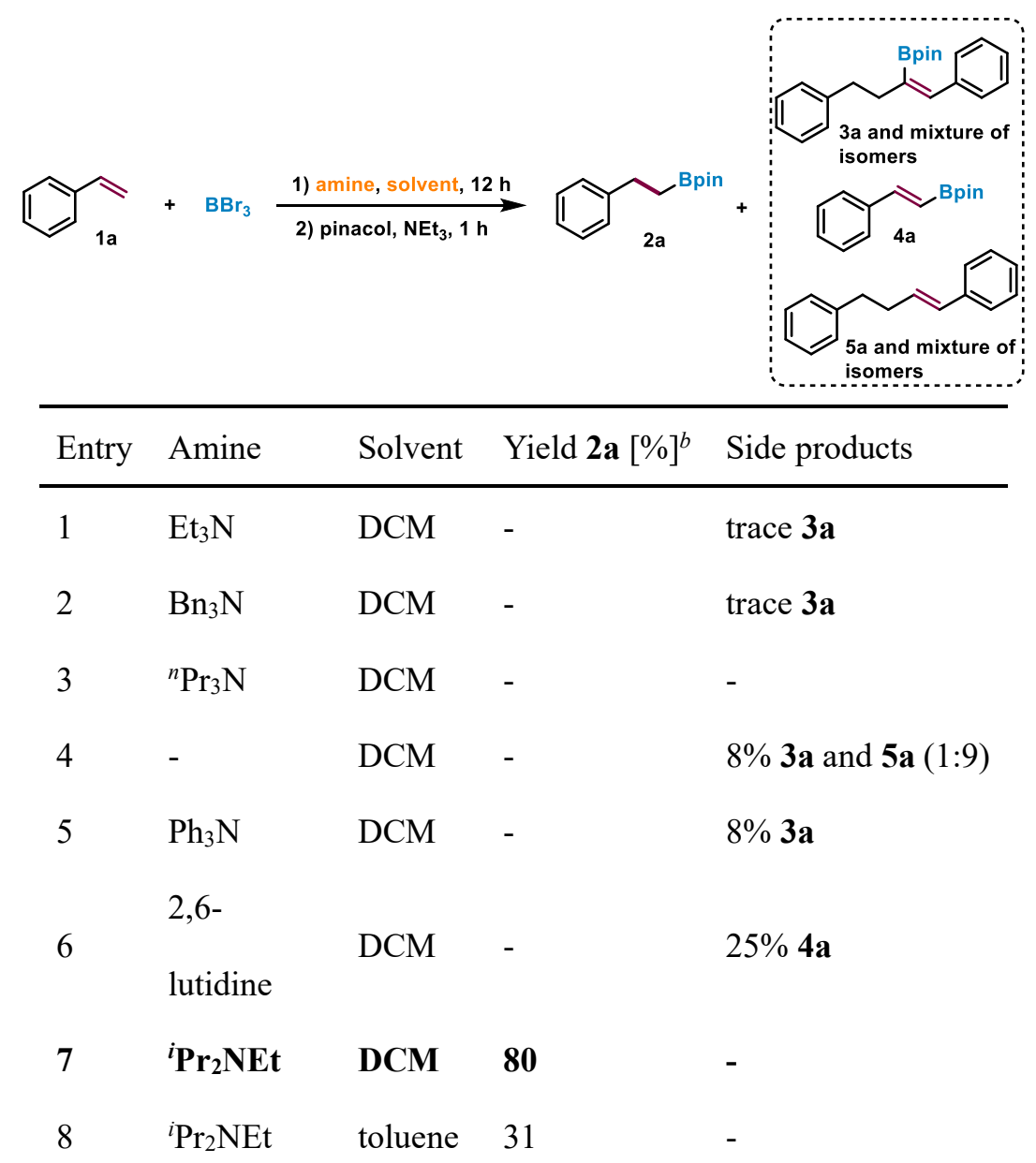




\begin{tabular}{lllll}
9 & ${ }^{i} \operatorname{Pr}_{2} \mathrm{NEt}$ & hexane & 26 & - \\
10 & ${ }^{i} \mathrm{Pr}_{2} \mathrm{NEt}$ & $\mathrm{DCE}$ & 12 & - \\
$11^{c}$ & ${ }^{i} \mathrm{Pr}_{2} \mathrm{NEt}$ & $\mathrm{DCM}$ & 23 & - \\
$12^{d}$ & ${ }^{i} \mathrm{Pr}_{2} \mathrm{NEt}$ & $\mathrm{DCM}$ & 75 & - \\
\hline
\end{tabular}

${ }^{a}$ Reaction conditions: $0.2 \mathrm{mmol} \mathbf{1 a}, \mathrm{BBr}_{3}$ (1.0 M in DCM, 1.5 equiv.), amines (3 equiv.), and $1 \mathrm{~mL} \mathrm{DCM}$ in a $5 \mathrm{~mL}$ glass crimp vial at $0{ }^{\circ} \mathrm{C}-\mathrm{rt}$ for $12 \mathrm{~h}$ and then pinacol and $\mathrm{Et}_{3} \mathrm{~N}$ were added and stirred for another $1 \mathrm{~h}$; ${ }^{b}$ yields of $\mathbf{2 a}$ were determined by GC/MS with dodecane as internal standard; ${ }^{c} \mathrm{BBr}_{3} \cdot \mathrm{SMe}_{2}$ was used; ${ }^{d} \mathrm{BCl}_{3}$ (1.0 M in DCM, 1.5 equiv.) was used.

Under the optimized reaction conditions (Table 1, entry 7), we then studied the substrates generality of our strategy. First, the reaction with terminal aryl alkenes was performed, we were pleased to find that our methodology worked well for a series of electronically and sterically varied aryl alkenes (Scheme 2). Substrates with $-\mathrm{Me},-{ }^{t} \mathrm{Bu},-\mathrm{CF}_{3}$, or $-\mathrm{Ph}$ groups at various positions on the phenyl ring reacted smoothly in our system with moderate to good yields $(\mathbf{2 a}-\mathbf{e}, \mathbf{2} \mathbf{i}, \mathbf{2 k}, 50-72 \%$ isolated yields) and $>97 \%$ regioselectivities. The use of halogen-substituted aryl alkenes selectively produced the corresponding hydroboration product without side reactions arising from $\mathrm{C}-\mathrm{X}$ bonds $(\mathbf{2 f}-\mathbf{h}, \mathbf{2} \mathbf{j}, \mathbf{2})$. In addition, vinyl naphthalene and pentafluorostyrene gave boronate esters $\mathbf{2 m}$ and 2n in yields of up to $82 \%$. Vinyl ferrocene and methyl(4-vinylphenyl)sulfane also reacted, albeit with lower yields $(\mathbf{2 0}, \mathbf{2 p})$.

The substrate scope with respect to 1,1-disubstituted aryl alkenes was then studied. In general, $\alpha$-methyl-substituted alkenes containing electronically varied aryl groups reacted smoothly, and yields up to $76 \%$ were obtained (2aa, 2ad-2af). Moreover, changing the $\alpha$-substituent from -Me to $-{ }^{n} \mathrm{Bu}$ and $-\mathrm{Ph}$ resulted in higher yields $(\mathbf{2} \mathbf{a b}, \mathbf{2} \mathbf{a c})$. In addition, alkenes with sterically varied cyclic aliphatic substituents reacted without problems (2ag-2ai). We then applied our protocol to longchain aliphatic alkenes and found that it worked with a series of bulk aliphatic alkenes with different carbon lengths (2ba-2bc) and substituents such as cyclohexyl-, and phenyl-substituted aliphatic alkenes (2bd-2bi). Noticed that, for bromide-substituted aliphatic alkenes, the -Br was kept intact (2bj-2bk). In addition, allyl boronate ester and alkenes containing sulfur-atom gave the corresponding product in up to $86 \%$ yields (2bl-2bm). Furthermore, bio-derived alkenes such as camphene or longifolene, and alkenes from 4'-tolyl-bicyclohexyl-4-one reacted well in our system with $>90 \%$ linear selectivity (2bn-2bp). 


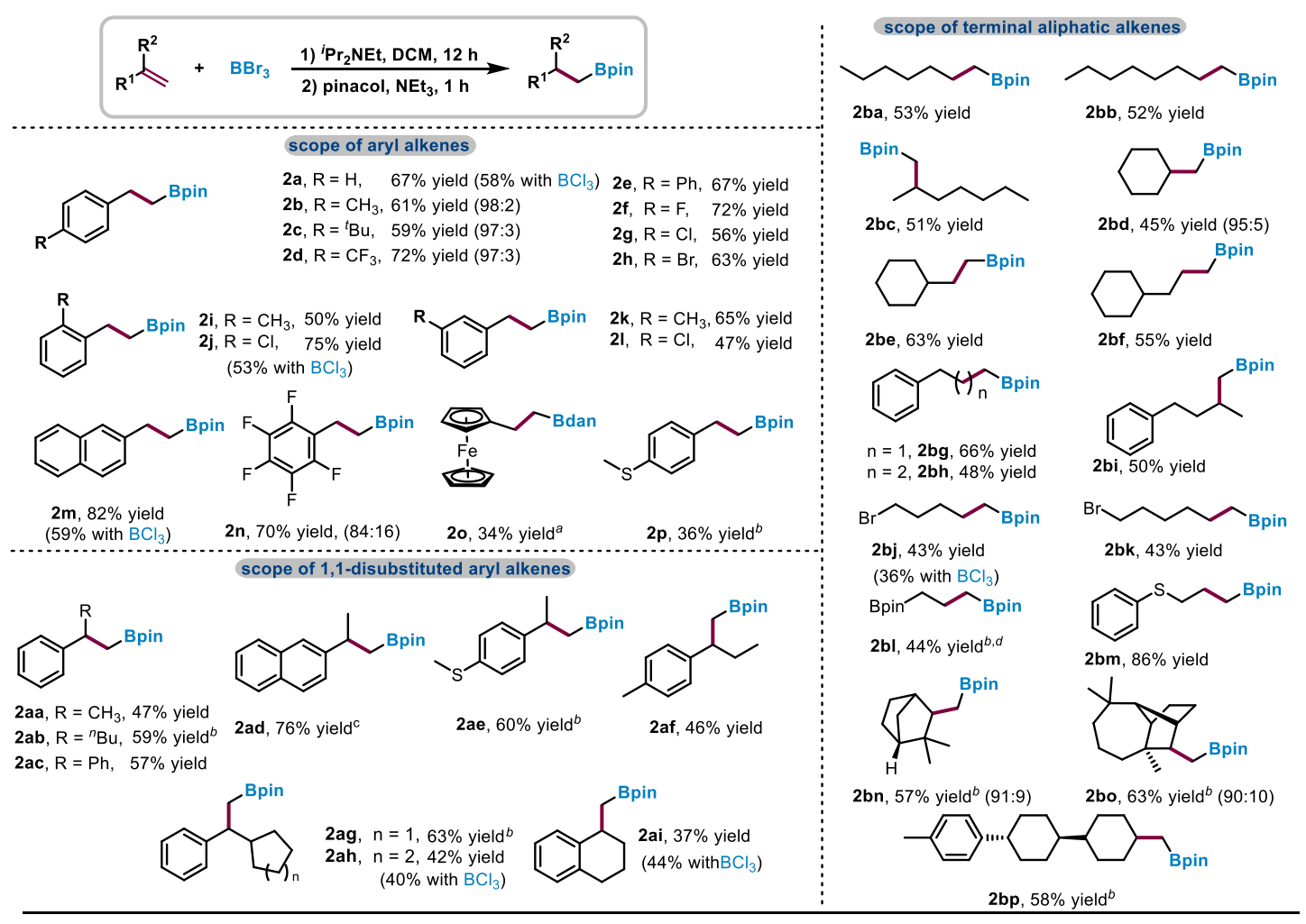

Scheme 2. Reaction conditions: $0.2 \mathrm{mmol}$ alkenes, $\mathrm{BBr}_{3}$ or $\mathrm{BCl}_{3}$ (1.0 M in DCM, 1.5 equiv.), ${ }^{i} \operatorname{Pr}_{2} \mathrm{NEt}(3$ equiv.), and $1 \mathrm{~mL}$ DCM in a $5 \mathrm{~mL}$ glass crimp vial at $0{ }^{\circ} \mathrm{C}-\mathrm{rt}$ for $12-24 \mathrm{~h}$ and then pinacol and $\mathrm{Et}_{3} \mathrm{~N}$ were added and stirred for another $1 \mathrm{~h}$, yields of isolated products are given. ${ }^{a}$ use 1,8-diaminonaphthalene instead of pinacol; ${ }^{b} 60{ }^{\circ} \mathrm{C}$ for $48 \mathrm{~h} ;{ }^{c} 80^{\circ} \mathrm{C}$ for $48 \mathrm{~h} ;{ }^{d} \mathrm{NMR}$ yield against anisole.

With the successful installation of the boronate unit at the terminal position of alkenes, we then further explored the possibility of applying our system for the site-fixed installation of a boronate unit at the original double-bond position of internal alkenes (Scheme 3). The reaction with 4-octene regioselectively produced the corresponding alkyl boronate $\mathbf{2} \mathbf{c a}$ in $58 \%$ yield. (E)-1,6-Diphenylhex3-ene underwent similar transformations without issue (2cb). In addition, for $N$-methyl-indolesubstituted internal alkenes with methyl or ethyl group attached to other side of the double-bonds, regioselective hydroboration took place at the $\beta$-position to the indole group $(\mathbf{2 c c}, \mathbf{2 c d})$; this was also the case when a longer carbon chain was attached (2ce). Moreover, sterically bulkier tris- and tetra-substituted internal alkenes were hydroborated without problems to give products $\mathbf{2 c f}$ and $\mathbf{2} \mathbf{c g}$, respectively, in up to $59 \%$ yield. The strategy was also applicable to cyclic internal alkenes such as cyclohexene and cycloheptene (2ch, 2ci). Interestingly, for cyclic vinyl boronate ester, selective boration at the $\alpha$-position of the bulky -Bpin group was observed (2cj). In addition, for bio-derived internal alkenes $\alpha$-cedrene, $67 \%$ of the corresponding boronate ester $\mathbf{2} \mathbf{c k}$ was obtained. Finally, to 
best demonstrate our strategy for site-fixed hydroboration of alkenes, substrates bearing doublebonds at different positions were applied, and the regioselective hydroboration along the carbon chain at 2-, 3-, 4-, and 5-positions was achieved (2cl-2co) (Scheme 3). It is also noteworthy to mention that several substrates were also tested using $\mathrm{BCl}_{3}$ as the boration reagent, though slightly lower yields were obtained, those results best demonstrated the application generality of our strategy and indicated a distinct mechanism difference with traditional electrophilic boration reactions. ${ }^{14 a-c}$

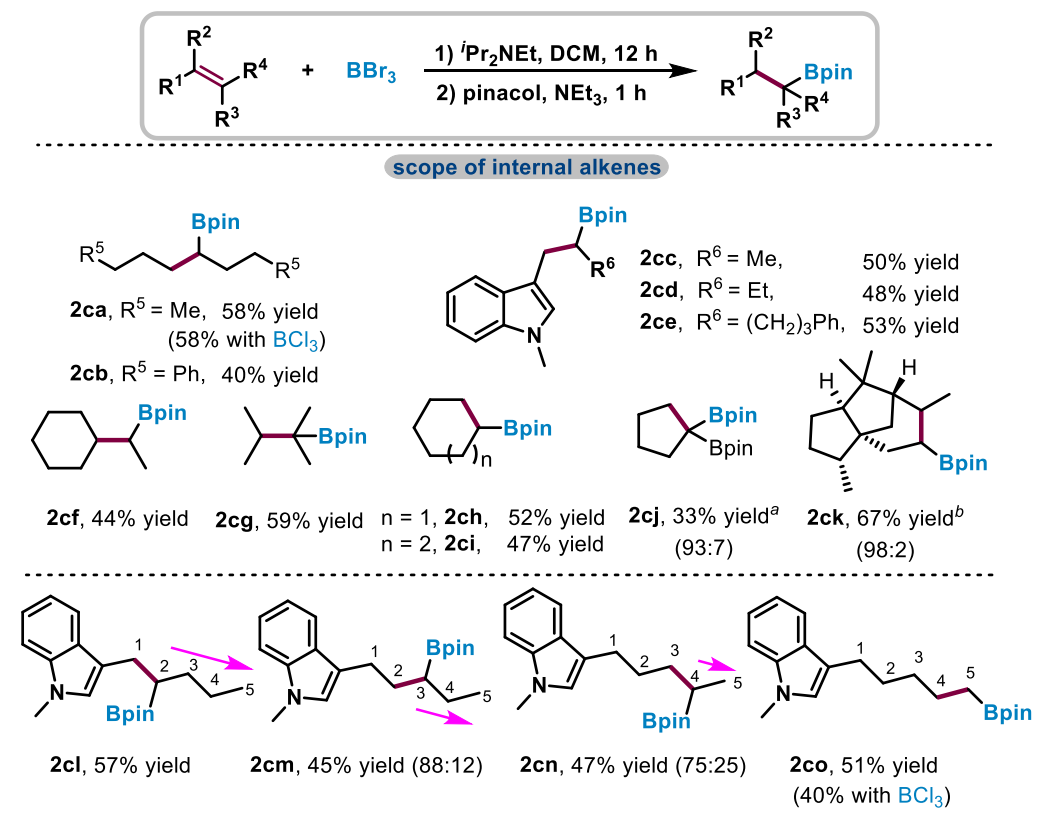

Scheme 3. Reaction conditions: $0.2 \mathrm{mmol}$ alkenes, $\mathrm{BBr}_{3}$ or $\mathrm{BCl}_{3}$ (1.0 M in DCM, 1.5 equiv.), ${ }^{i} \mathrm{Pr}_{2} \mathrm{NEt}(3$ equiv.), and $1 \mathrm{~mL}$ DCM in a $5 \mathrm{~mL}$ glass crimp vial at $0{ }^{\circ} \mathrm{C}-\mathrm{rt}$ for $12-48 \mathrm{~h}$ and then pinacol and $\mathrm{Et}_{3} \mathrm{~N}$ were added and stirred for another $1 \mathrm{~h}$, yields of isolated products are given. ${ }^{a} 60{ }^{\circ} \mathrm{C}$ for $48 \mathrm{~h} ;{ }^{b} 80{ }^{\circ} \mathrm{C}$ for $48 \mathrm{~h}$.

To understand the reaction mechanism, several control experiments were conducted. First, when radical inhibitors such as 2,2,6,6-tetramethylpiperidine 1-oxyl (TEMPO) or 2,6-di-tert-butyl4-[(3,5-di-tert-butyl-4- $\lambda 1$-oxidanylphenyl)methylidene]cyclohexa-2,5-dien-1-one (galvinoxyl) were added, inhibition of the reaction was observed (Scheme 4a). A complete lack of electron paramagnetic resonance (EPR) signal from the reaction mixture was also noted with the addition of TEMPO (Scheme 4b). Furthermore, by adding 5,5-dimethyl-1-pyrroline $N$-oxide (DMPO) (0.5 equiv.) into a solution of $\mathrm{BBr}_{3} / \mathrm{Pr}_{2} \mathrm{NEt}$ or $\mathrm{BBr}_{3} /{ }^{2} \mathrm{Pr}_{2} \mathrm{NEt} / \mathrm{styrene,} \mathrm{EPR} \mathrm{active} \mathrm{signals} \mathrm{were} \mathrm{detected,}$ which implies the formation of radical species (Scheme 4c). The involvement of radical species was also confirmed using $\mathbf{1 a k}$ as a radical clock reaction substrate; in this case, the ring-opening product $\mathbf{2} \mathbf{a k}^{\prime}$ was detected in addition to the hydroboration product 2ak (Scheme 4d). To assess the potential 
electronic effect of the radical process, the reaction of an electronically different set of $p$-substituted styrene with $\mathrm{BBr}_{3}$ was carried out. A Hammett plot was constructed, the results revealed a linear correlation between the $\log \left(\mathrm{k}_{\mathrm{X}} / \mathrm{k}_{\mathrm{H}}\right)$ and the Hammett constants $\left(\sigma_{p}\right)$ of the para-substituents with a negative slope of -0.59 . The Hammett plot signifies the electrophilic nature of the radical intermediate (Scheme 4e). When $\mathrm{D}_{8}$-styrene was reacted with $\mathrm{BBr}_{3}$ in the presence of ${ }^{i} \mathrm{Pr}_{2} \mathrm{NEt}, 44 \%$ $\mathrm{H}$-incorporation into the $\beta$-position of $\mathbf{2 a}$ was identified (Scheme 4f). The possible $\mathrm{H}$-atom source should be ${ }^{i} \mathrm{Pr}_{2} \mathrm{NEt}$ or dichloromethane. Thus, the reaction of styrene in $\mathrm{D}_{2}$-dichloromethane was conducted, and we found no D-incorporation in product 2a (Scheme 4g). Furthermore, analyzing the reaction solution of mixed $\mathrm{BBr}_{3} /{ }^{i} \mathrm{Pr}_{2} \mathrm{NEt}$ by ${ }^{13} \mathrm{C}$ NMR spectroscopy, two peaks at 187.8 and $152.6 \mathrm{ppm}$ signaled the possible presence of iminium species I-1 or I-2 (Scheme 4h). Altogether, the deuterium-labeling experiments and the formation of iminium species indicated that the $\mathrm{H}$-atom came from ${ }^{i} \mathrm{Pr}_{2} \mathrm{NEt}$. Finally, the presence of iminium species was further confirmed by ESI-MS analysis (Scheme 4i). 
a)

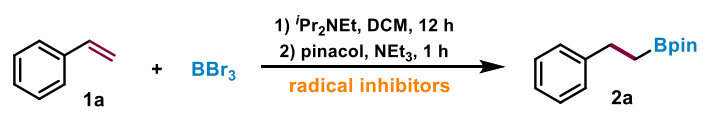

b)

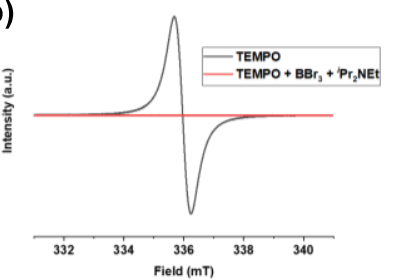

d)

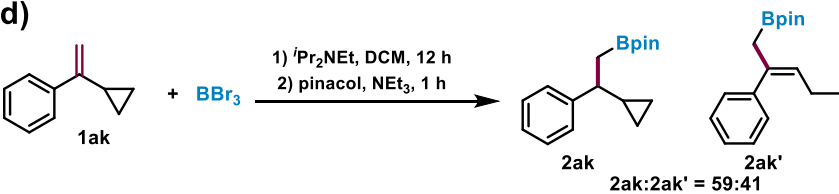

e)

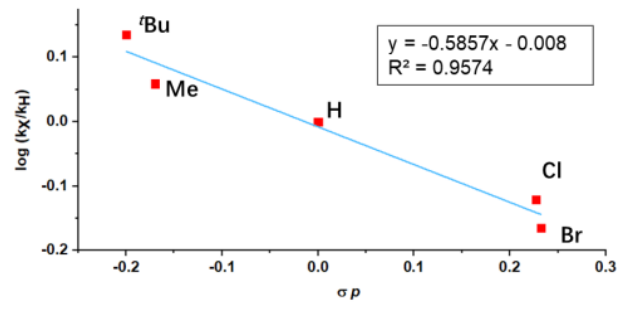

f)

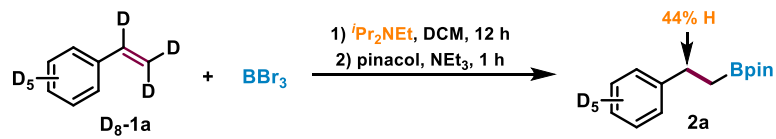

g)

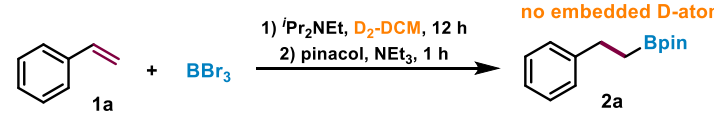

h)
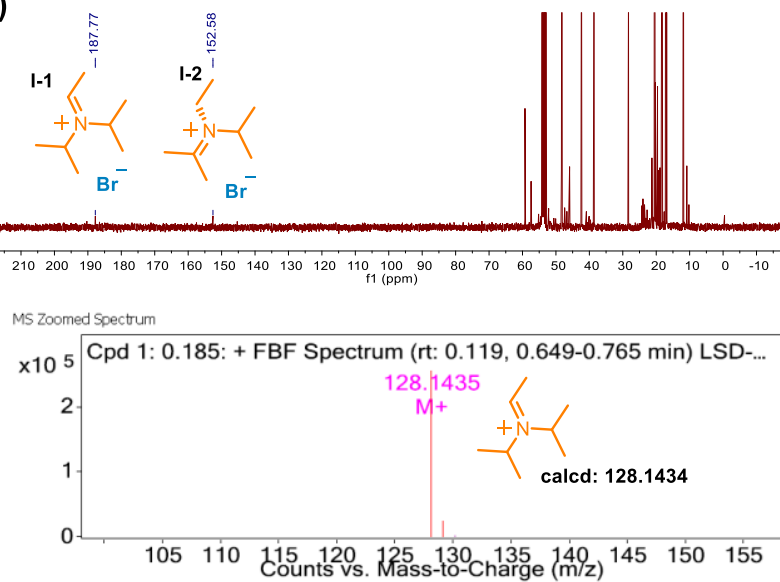

c)

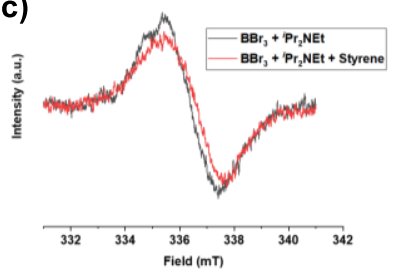

$2 a$

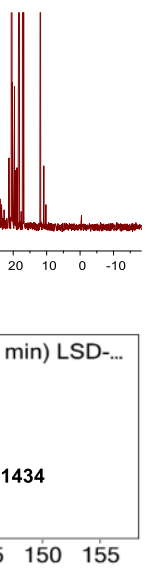

Scheme 4. Mechanism studies: a) addition of different radical inhibitor into the standard reaction; b) EPR spectra of $0.1 \mathrm{mmol}$ tempo with (-) and without (-) $0.3 \mathrm{mmol}$ of $\mathrm{BBr}_{3}$ and $0.3 \mathrm{mmol}{ }^{i} \mathrm{Pr}_{2} \mathrm{NEt}$ in a mixture of $1 \mathrm{~mL}$ of DCM and $1 \mathrm{~mL}$ of toluene; c) EPR spectra of $0.1 \mathrm{mmol}$ of DMPO with $0.3 \mathrm{mmol}$ of $\mathrm{BBr}_{3}$ and $0.6 \mathrm{mmol}^{i} \operatorname{Pr}_{2} \mathrm{NEt}$ in a mixture of $1 \mathrm{~mL}$ of DCM and $1 \mathrm{~mL}$ of toluene with (-) and without (-) $0.2 \mathrm{mmol}$ styrene; d) radical clock reaction with substrates 1ak at $0.2 \mathrm{mmol}$ scales under standard reaction conditions; e) Hammett plot of different set of $p$ substituted styrene; f) deuterium-labeling experiments with $\mathrm{D}_{8}$-styrene; g) standard reaction with styrene and use $\mathrm{D}_{2}$-dichloromethane as solvent; h) ${ }^{13} \mathrm{C}$ NMR spectrum of $0.3 \mathrm{mmol} \mathrm{BBr}_{3}$ and $0.6 \mathrm{mmol}{ }^{i} \mathrm{Pr}_{2} \mathrm{NEt}$ in $1 \mathrm{~mL}$ of $\mathrm{D}_{2}$ dichloromethane; i) ESI-MS spectrum of $0.3 \mathrm{mmol} \mathrm{BBr}_{3}$ and $0.6 \mathrm{mmol}{ }^{i} \mathrm{Pr}_{2} \mathrm{NEt}$ with $0.2 \mathrm{mmol}$ styrene in $1 \mathrm{~mL}$ of dichloromethane. 
Based on the abovementioned observations and recent reports on single-electron transfer of FLP chemistry ${ }^{19}$ and boryl radical formations, ${ }^{21}$ a tentative reaction pathway involving the formation of radical species was proposed (Scheme 5). First, complexation of $\mathrm{BBr}_{3}$ with ${ }^{i} \mathrm{Pr}_{2} \mathrm{NEt}$ forms a Lewis acid-base adduct $\mathbf{A B}$. The reversible formation of $\mathbf{A B}$ at room temperature was crucial for the single-electron transfer FLP chemistry to take place, which was confirmed by ${ }^{11} \mathrm{~B}$ NMR spectroscopy studies (Figure S7). Then single-electron transfer between ${ }^{i} \operatorname{Pr}_{2} \mathrm{NEt}$ and $\mathrm{BBr}_{3}$ by transferring one electron from ${ }^{i} \mathrm{Pr}_{2} \mathrm{NEt}$ to form the amine radical cation and $\mathrm{BBr}_{3}$ radical anion. Subsequent cleavage of $\mathrm{BBr}_{3}{ }^{--}$radical anion forms a $\mathrm{BBr}_{2}{ }^{\cdot}$ radical and $\mathrm{Br}^{-}$, the presence of $\mathrm{Br}^{-}$was also confirmed by the ${ }^{11} \mathrm{~B}$ NMR signal of $\mathrm{BBr}_{4}^{-}$(Figure S8). The $\mathrm{BBr}_{2}{ }^{\cdot}$ radical then adds to the double bond of the alkene to form a new alkyl radical, which abstracts a H-radical from the amine radical cation to give the final hydroboration product.

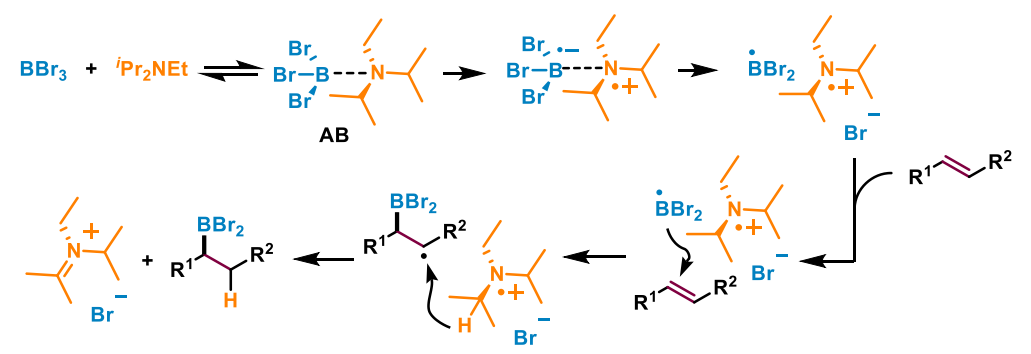

Scheme 5. Tentatively proposed reaction pathway.

In summary, we have developed a transition-metal-free alkene hydroboration system simply using $\mathrm{BBr}_{3}$ as the boration reagent in the presence of ${ }^{i} \mathrm{Pr}_{2} \mathrm{NEt}$. The addition of ${ }^{i} \mathrm{Pr}_{2} \mathrm{NEt}$ not only suppresses the alkene oligomerization and bromoboration side reactions but also plays a key role in the hydroboration reaction by providing a proton source. A series of alkenes, including aryl alkene, 1,1-disubstituted alkenes, aliphatic alkenes, and bio-derived alkenes were readily hydroborated. More importantly, the site-fixed installation of the boryl group at the original position of the internal alkene was also realized, which is otherwise difficult to achieve using traditional transition-metal catalysts. Studies on the mechanism revealed a reaction pathway that might operate through FLPtype single-electron transfer.

\section{Acknowledgement}

We are grateful to the National Natural Science Foundation of China (91845108, 21901247, 21902167), Natural Science Foundation of Jiangsu Province (BK20180246), and the "Innovation \& 
Entrepreneurship Talents Plan" of Jiangsu Province for generous financial support. We would also like to thank the National Program for Young Investigator of China for support to start the Lab.

\section{Data availability}

All data are available upon request.

\section{References}

1. (a) Miyaura, N.; Suzuki, A., Palladium-Catalyzed Cross-Coupling Reactions of Organoboron Compounds. Chem. Rev. 1995, 95 (7), 2457-2483; (b) Lennox, A. J.; Lloyd-Jones, G. C., Selection of boron reagents for Suzuki-Miyaura coupling. Chem. Soc. Rev. 2014, 43 (1), 412-43; (c) Bonet, A.; Odachowski, M.; Leonori, D.; Essafi, S.; Aggarwal, V. K., Enantiospecific sp2-sp3 coupling of secondary and tertiary boronic esters. Nat. Chem. 2014, 6, 584; (d) Xu, L.; Zhang, S.; Li, P., Boron-selective reactions as powerful tools for modular synthesis of diverse complex molecules. Chem. Soc. Rev. 2015, 44 (24), 8848-58; (e) Neeve, E. C.; Geier, S. J.; Mkhalid, I. A.; Westcott, S. A.; Marder, T. B., Diboron(4) Compounds: From Structural Curiosity to Synthetic Workhorse. Chem. Rev. 2016, 116 (16), 9091-161;

(f) Fawcett, A.; Nitsch, D.; Ali, M.; Bateman, J. M.; Myers, E. L.; Aggarwal, V. K., Regio- and Stereoselective Homologation of 1,2-Bis(Boronic Esters): Stereocontrolled Synthesis of 1,3-Diols and Sch 725674. Angew. Chem. Int. Ed. 2016, 55 (47), 14663-14667; (g) Smith, J. R.; Collins, B. S. L.; Hesse, M. J.; Graham, M. A.; Myers, E. L.; Aggarwal, V. K., Enantioselective Rhodium(III)-Catalyzed Markovnikov Hydroboration of Unactivated Terminal Alkenes. J. Am. Chem. Soc. 2017, 139 (27), 9148-9151; (h) Wu, J.; He, L.; Noble, A.; Aggarwal, V. K., Photoinduced Deaminative Borylation of Alkylamines. J. Am. Chem. Soc. 2018, 140 (34), 10700-10704; (i) Namirembe, S.; Morken, J. P., Reactions of organoboron compounds enabled by catalyst-promoted metalate shifts. Chem. Soc. Rev. 2019, 48 (13), 3464-3474.

2. (a) Hall, D. G., Boronic Acids: Preparation and Applications in Organic Synthesis and Medicine. Ed.; Wiley-VCH: Weinheim, Germany, 2005; (b) Cheng, F.; Jäkle, F., Boron-containing polymers as versatile building blocks for functional nanostructured materials. Polym. Chem. 2011, 2 (10), 2122-2132; (c) Kim, J.; Park, S.; Park, J.; Cho, S. H., Synthesis of Branched Alkylboronates by Copper-Catalyzed Allylic Substitution Reactions of Allylic Chlorides with 1,1-Diborylalkanes. Angew. Chem. Int. Ed. 2016, 55 (4), 1498-1501; (d) Diaz, D. B.; Yudin, A. K., The versatility of boron in biological target engagement. Nat. Chem. 2017, 9 (8), 731-742; (e) Kim, J.; Ko, K.; Cho, S. H., Diastereo- and Enantioselective Synthesis of $\beta$-Aminoboronate Esters by Copper(I)-Catalyzed 1,2-Addition of 1,1-Bis[(pinacolato)boryl]alkanes to Imines. Angew. Chem. Int. Ed. 2017, 56 (38), 11584-11588; (f) Chauhan, N. P. S.; Hosmane, N. S.; Mozafari, M., Boron-based polymers: opportunities and challenges. Mater. Today Chem. 2019, 


\section{4, 100184.}

3. (a) Bonet, A.; Pubill-Ulldemolins, C.; Bo, C.; Gulyás, H.; Fernández, E., Transition-Metal-Free Diboration Reaction by Activation of Diboron Compounds with Simple Lewis Bases. Angew. Chem. Int. Ed. 2011, 50 (31), 7158-7161; (b) Takaya, J.; Iwasawa, N., Catalytic, Direct Synthesis of Bis(boronate) Compounds. ACS. Catal. 2012, 2 (9), 1993-2006; (c) Coombs, J. R.; Zhang, L.; Morken, J. P., Enantiomerically enriched tris(boronates): readily accessible conjunctive reagents for asymmetric synthesis. J. Am. Chem. Soc. 2014, 136 (46), 16140-3; (d) Hong, K.; Liu, X.; Morken, J. P., Simple access to elusive alpha-boryl carbanions and their alkylation: an umpolung construction for organic synthesis. J. Am. Chem. Soc. 2014, 136 (30), 10581-4; (e) Potter, B.; Szymaniak, A. A.; Edelstein, E. K.; Morken, J. P., Nonracemic allylic boronates through enantiotopic-group-selective cross-coupling of geminal bis(boronates) and vinyl halides. J. Am. Chem. Soc. 2014, 136 (52), 17918-21; (f) Jang, W. J.; Lee, W. L.; Moon, J. H.; Lee, J. Y.; Yun, J., Copper-Catalyzed trans-Hydroboration of Terminal Aryl Alkynes: Stereodivergent Synthesis of Alkenylboron Compounds. Org. Lett. 2016, 18 (6), 1390-3; (g) Fyfe, J. W. B.; Watson, A. J. B., Recent Developments in Organoboron Chemistry: Old Dogs, New Tricks. Chem 2017, 3 (1), 31-55; (h) Li, L.; Gong, T.; Lu, X.; Xiao, B.; Fu, Y., Nickel-catalyzed synthesis of 1,1-diborylalkanes from terminal alkenes. Nat. Commun. 2017, 8 (1), 345; (i) Yan, G.; Huang, D.; Wu, X., Recent Advances in C-B Bond Formation through a Free Radical Pathway. Adv. Synth. Catal. 2018, 360 (6), 1040-1053; (j) Hu, J.; Zhao, Y.; Shi, Z., Highly tunable multiborylation of gem-difluoroalkenes via copper catalysis. Nat. Catal. 2018, 1 (11), 860-869; (k) Teo, W. J.; Ge, S., Cobalt-Catalyzed Diborylation of 1,1-disubstituted Vinylarenes: A Practical Route to Branched gemBis(boryl)alkanes. Angew. Chem. Int. Ed. 2018, 57 (6), 1654-1658; (1) Yamazaki, A.; Nagao, K.; Iwai, T.; Ohmiya, H.; Sawamura, M., Phosphine-Catalyzed anti-Carboboration of Alkynoates with 9-BBN-Based 1,1-Diborylalkanes: Synthesis and Use of Multisubstituted $\gamma$-Borylallylboranes. Angew. Chem. Int. Ed. 2018, 57 (12), 3196-3199; (m) Zhao, B.; Li, Z.; Wu, Y.; Wang, Y.; Qian, J.; Yuan, Y.; Shi, Z., An Olefinic 1,2-Boryl-Migration Enabled by Radical Addition: Construction of gem-Bis(boryl)alkanes. Angew. Chem. Int. Ed. 2019, 58 (28), 9448-9452; (n) Maza, R. J.; Davenport, E.; Miralles, N.; Carbo, J. J.; Fernandez, E., Transition-Metal-Free Allylic Borylation of 1,3-Dienes. Org. Lett. 2019, 21 (7), 2251-2255; (o) Lee, H.; Lee, S.; Yun, J., Pd-Catalyzed Stereospecific Cross-Coupling of Chiral a-Borylalkylcopper Species with Aryl Bromides. ACS. Catal. 2020, 10 (3), 2069-2073; (p) Reyes, R. L.; Sato, M.; Iwai, T.; Suzuki, K.; Maeda, S.; Sawamura, M., Asymmetric remote C-H borylation of aliphatic amides and esters with a modular iridium catalyst. Science 2020, 369 (6506), 970-974.

4. (a) Ito, H.; Kubota, K., Copper(I)-catalyzed boryl substitution of unactivated alkyl halides. Org. Lett. 2012, 14 (3), 890-3; (b) Chow, W. K.; Yuen, O. Y.; Choy, P. Y.; So, C. M.; Lau, C. P.; Wong, W. T.; Kwong, F. Y., A decade 
advancement of transition metal-catalyzed borylation of aryl halides and sulfonates. $R S C A d v . \mathbf{2 0 1 3}, 3$ (31), 1251812539; (c) Cuenca, A. B.; Shishido, R.; Ito, H.; Fernández, E., Transition-metal-free B-B and B-interelement reactions with organic molecules. Chem. Soc. Rev. 2017, 46 (2), 415-430.

5. (a) Mkhalid, I. A. I.; Barnard, J. H.; Marder, T. B.; Murphy, J. M.; Hartwig, J. F., C-H Activation for the Construction of C-B Bonds. Chem. Rev. 2010, 110 (2), 890-931; (b) Ros, A.; Fernández, R.; Lassaletta, J. M., Functional group directed C-H borylation. Chem. Soc. Rev. 2014, 43 (10), 3229-3243.

6. (a) Chen, D.; Zhang, X.; Qi, W.-Y.; Xu, B.; Xu, M.-H., Rhodium(I)-Catalyzed Asymmetric Carbene Insertion into B-H Bonds: Highly Enantioselective Access to Functionalized Organoboranes. J. Am. Chem. Soc. 2015, 137 (16), 5268-5271; (b) Yang, J.-M.; Li, Z.-Q.; Li, M.-L.; He, Q.; Zhu, S.-F.; Zhou, Q.-L., Catalytic B-H Bond Insertion Reactions Using Alkynes as Carbene Precursors. J. Am. Chem. Soc. 2017, 139 (10), 3784-3789.

7. (a) Burgess, K.; Ohlmeyer, M. J., Transition-metal promoted hydroborations of alkenes, emerging methodology for organic transformations. Chem. Rev. 1991, 91 (6), 1179-1191; (b) Obligacion, J. V.; Chirik, P. J., Earth-abundant transition metal catalysts for alkene hydrosilylation and hydroboration. Nat. Rev. Chem. 2018, 2 (5), 15-34.

8. Brown, H. C.; Zweifel, G., Hydroboration. VII. Directive Effects in the Hydroboration of Olefins. J. Am. Chem. Soc. 1960, 82 (17), 4708-4712.

9. (a) Brown, H. C.; Gupta, S. K., Hydroboration. XXXIX. 1,3,2-Benzodioxaborole (catecholborane) as a new hydroboration reagent for alkenes and alkynes. General synthesis of alkane- and alkeneboronic acids and esters via hydroboration. Directive effects in the hydroboration of alkenes and alkynes with catecholborane. J. Am. Chem. Soc. 1975, 97 (18), 5249-5255; (b) Kabalka, G. W., Catecholborane in Organic Synthesis. A Review. Org. Prep. Proced. Int. 1977, 9 (3), 131-147.

10. Lee, Y.; Hoveyda, A. H., Efficient Boron-Copper Additions to Aryl-Substituted Alkenes Promoted by NHC-Based Catalysts. Enantioselective Cu-Catalyzed Hydroboration Reactions. J. Am. Chem. Soc. 2009, 131 (9), 3160-3161.

11. (a) Xi, Y.; Hartwig, J. F., Diverse Asymmetric Hydrofunctionalization of Aliphatic Internal Alkenes through Catalytic Regioselective Hydroboration. J. Am. Chem. Soc. 2016, 138 (21), 6703-6706; (b) Xi, Y.; Hartwig, J. F., Mechanistic Studies of Copper-Catalyzed Asymmetric Hydroboration of Alkenes. J. Am. Chem. Soc. 2017, 139 (36), $12758-12772$.

12. (a) Feng, X.; Jeon, H.; Yun, J., Regio- and enantioselective copper(I)-catalyzed hydroboration of borylalkenes: asymmetric synthesis of 1,1-diborylalkanes. Angew. Chem. Int. Ed. 2013, 52 (14), 3989-92; (b) Noh, D.; Yoon, S. K.; Won, J.; Lee, J. Y.; Yun, J., An Efficient Copper(I)-Catalyst System for the Asymmetric Hydroboration of $\beta$ - 
Substituted Vinylarenes with Pinacolborane. Chem. Asian J. 2011, 6 (8), 1967-1969.

13. Major, C. J.; Bamford, K. L.; Qu, Z.-W.; Stephan, D. W., Hydroboration without a B-H bond: reactions of the borinium cation $\left[\left(\mathrm{iPr}_{2} \mathrm{~N}\right)_{2} \mathrm{~B}\right]+$ with alkyne, nitrile, ketone and diazomethane. Chem. Commun. 2019, 55 (35), $5155-$ 5158.

14. (a) Yang, C.-H.; Zhang, Y.-S.; Fan, W.-W.; Liu, G.-Q.; Li, Y.-M., Intramolecular Aminoboration of Unfunctionalized Olefins. Angew. Chem. Int. Ed. 2015, 54 (43), 12636-12639; (b) Warner, A. J.; Lawson, J. R.; Fasano, V.; Ingleson, M. J., Formation of C(sp2)-Boronate Esters by Borylative Cyclization of Alkynes Using $\mathrm{BCl}_{3}$. Angew. Chem. Int. Ed. 2015, 54 (38), 11245-11249; (c) Warner, A. J.; Churn, A.; McGough, J. S.; Ingleson, M. J., BCl3-Induced Annulative Oxo- and Thioboration for the Formation of C3-Borylated Benzofurans and Benzothiophenes. Angew. Chem. Int. Ed. 2017, 56 (1), 354-358; (d) Lv, J.; Zhao, B.; Liu, L.; Han, Y.; Yuan, Y.; Shi, Z., Boron Trichloride-Mediated Synthesis of Indoles via the Aminoboration of Alkynes. Adv. Synth. Catal. 2018, $360(21), 4054-4059$.

15. (a) Lv, J.; Chen, X.; Xue, X.-S.; Zhao, B.; Liang, Y.; Wang, M.; Jin, L.; Yuan, Y.; Han, Y.; Zhao, Y.; Lu, Y.; Zhao, J.; Sun, W.-Y.; Houk, K. N.; Shi, Z., Metal-free directed sp2-C-H borylation. Nature 2019, 575 (7782), 336-340; (b) Iqbal, S. A.; Cid, J.; Procter, R. J.; Uzelac, M.; Yuan, K.; Ingleson, M. J., Acyl-Directed ortho-Borylation of Anilines and C7 Borylation of Indoles using just $\mathrm{BBr}_{3}$. Angew. Chem. Int. Ed. 2019, 58 (43), 15381-15385.

16. (a) Lappert, M. F.; Prokai, B., Chloroboration and allied reactions of unsaturated compounds II. Haloboration and phenylboration of acetylenes; and the preparation of some alkynylboranes. J. Organomet. Chem. 1964, 1 (5), 384-400; (b) Satoh, Y.; Serizawa, H.; Miyaura, N.; Hara, S.; Suzuki, A., Organic synthesis using haloboration reactions 11. A formal carboboration reaction of 1-alkynes and its application to the di- and trisubstituted alkene synthesis. Tetrahedron Lett. 1988, 29 (15), 1811-1814.

17. Soundararajan, R.; Matteson, D. S., Hydroboration with Haloborane/Trialkylsilane Mixtures. Organometallics 1995, 14 (9), 4157-4166.

18. Tanaka, S.; Saito, Y.; Yamamoto, T.; Hattori, T., Electrophilic Borylation of Terminal Alkenes with $\mathrm{BBr}_{3} / 2,6-$ Disubstituted Pyridines. Org. Lett. 2018, 20 (7), 1828-1831.

19. (a) Liu, L.; Cao, L. L.; Shao, Y.; Ménard, G.; Stephan, D. W., A Radical Mechanism for Frustrated Lewis Pair Reactivity. Chem 2017, 3 (2), 259-267; (b) Liu, L. L.; Cao, L. L.; Shao, Y.; Stephan, D. W., Single Electron Delivery to Lewis Pairs: An Avenue to Anions by Small Molecule Activation. J. Am. Chem. Soc. 2017, 139 (29), 10062-10071; (c) Holtrop, F.; Jupp, A. R.; Kooij, B. J.; van Leest, N. P.; de Bruin, B.; Slootweg, J. C., Single-Electron Transfer in Frustrated Lewis Pair Chemistry. Angew. Chem. Int. Ed. 2020, 59 (49), 22210-22216; (d) Liu, L. L.; Stephan, D. W., 
Radicals derived from Lewis acid/base pairs. Chem. Soc. Rev. 2019, 48 (13), 3454-3463; (e) Ayan, D.; Richards, E.; Melen, R., Frustrated Radical Pairs: Insights from EPR Spectroscopy. Angew. Chem. Int. Ed. 2021, 60 (1), 53-65; (f) Jupp, A. R.; Stephan, D. W., New Directions for Frustrated Lewis Pair Chemistry. Trends Chem. 2019, 1 (1), 35-48; (g) Aramaki, Y.; Imaizumi, N.; Hotta, M.; Kumagai, J.; Ooi, T., Exploiting single-electron transfer in Lewis pairs for catalytic bond-forming reactions. Chem. Sci. 2020, 11 (17), 4305-4311; (h) Soltani, Y.; Dasgupta, A.; Gazis, T. A.; Ould, D. M. C.; Richards, E.; Slater, B.; Stefkova, K.; Vladimirov, V. Y.; Wilkins, L. C.; Willcox, D.; Melen, R. L., Radical Reactivity of Frustrated Lewis Pairs with Diaryl Esters. Cell Rep. Phys. Sci. 2020, 1 (2), 100016.

20. (a) Kessar, S. V.; Singh, P., Lewis Acid Complexation of Tertiary Amines and Related Compounds: A Strategy for $\alpha$-Deprotonation and Stereocontrol. Chem. Rev. 1997, 97 (3), 721-738; (b) Voss, T.; Mahdi, T.; Otten, E.; Fröhlich, R.; Kehr, G.; Stephan, D. W.; Erker, G., Frustrated Lewis Pair Behavior of Intermolecular Amine/B( $\left.\mathrm{C}_{6} \mathrm{~F}_{5}\right)_{3}$ Pairs. Organometallics 2012, 31 (6), 2367-2378.

21. (a) Ueng, S.-H.; Solovyev, A.; Yuan, X.; Geib, S. J.; Fensterbank, L.; Lacôte, E.; Malacria, M.; Newcomb, M.; Walton, J. C.; Curran, D. P., N-Heterocyclic Carbene Boryl Radicals: A New Class of Boron-Centered Radical. J. Am. Chem. Soc. 2009, 131 (31), 11256-11262; (b) Walton, J. C.; Brahmi, M. M.; Fensterbank, L.; Lacôte, E.; Malacria, M.; Chu, Q.; Ueng, S.-H.; Solovyev, A.; Curran, D. P., EPR Studies of the Generation, Structure, and Reactivity of N-Heterocyclic Carbene Borane Radicals. J. Am. Chem. Soc. 2010, 132 (7), 2350-2358; (c) Taniguchi, T., Boryl Radical Addition to Multiple Bonds in Organic Synthesis. Eur. J. Org. Chem. 2019, 2019 (37), 6308-6319. 